University of South Florida

DIGITAL COMMONS

Digital Commons @ University of

@ UNIVERSITY OF SOUTH FLORIDA

South Florida

March 2020

\title{
An Examination of Changes in Muscle Thickness, Isometric Strength, and Body Water Throughout the Menstrual Cycle
}

Tayla E. Kuehne

University of South Florida

Follow this and additional works at: https://digitalcommons.usf.edu/etd

Part of the Other Education Commons, and the Physiology Commons

\section{Scholar Commons Citation}

Kuehne, Tayla E., "An Examination of Changes in Muscle Thickness, Isometric Strength, and Body Water Throughout the Menstrual Cycle" (2020). USF Tampa Graduate Theses and Dissertations.

https://digitalcommons.usf.edu/etd/8237

This Thesis is brought to you for free and open access by the USF Graduate Theses and Dissertations at Digital Commons @ University of South Florida. It has been accepted for inclusion in USF Tampa Graduate Theses and Dissertations by an authorized administrator of Digital Commons @ University of South Florida. For more information, please contact digitalcommons@usf.edu. 
An Examination of Changes in Muscle Thickness, Isometric Strength, and Body Water Throughout the Menstrual Cycle

By

Tayla E. Kuehne

\author{
A thesis submitted in partial fulfillment \\ of the requirements for the degree of \\ Master of Science \\ Department of Educational and Psychological Studies \\ College of Education \\ University of South Florida
}

Major Professor: Samuel L. Buckner, PhD

Nicholas Martinez, $\mathrm{PhD}$

Bill Campbell, $\mathrm{PhD}$

Date of Approval:

March 13, 2020

Keywords: Bioelectrical Impedance, Ovulation, B-Mode Ultrasound, Acute Swelling Copyright (C) 2020, Tayla E. Kuehne 


\section{Table of Contents}

LIST OF TABLES

LIST OF FIGURES

ABSTRACT v

CHAPTER 1: INTRODUCTION

Rationale

Problem Statement

3

Research Variables $\quad 4$

Hypotheses $\quad 4$

Operational Definitions $\quad 4$

Assumptions $\quad 5$

$\begin{array}{ll}\text { Limitations } & 6\end{array}$

Delimitations $\quad 6$

Significance $\quad 7$

CHAPTER 2: REVIEW OF LITERATURE

Introduction $\quad 8$

Background $\quad 8$

Menstrual Cycle and Strength 9

Menstrual Cycle and Muscle Size $\quad 11$

Muscle Thickness $\quad 12$

Muscle Swelling $\quad 12$

CHAPTER 3: METHODOLOGY

Participants $\quad 14$

Study Inclusion/Exclusion Criteria $\quad 14$

Study Overview $\quad 15$

Measurements 15

$\begin{array}{ll}\text { Equipment } & 17\end{array}$

$\begin{array}{ll}\text { Procedures } & 18\end{array}$

$\begin{array}{lr}\text { Statistical Analysis } & 20\end{array}$

CHAPTER 4: RESULTS

Demographics $\quad 21$

Total Body Water $\quad 21$

Isometric Strength $\quad 22$ 
Muscle Thickness

Muscle Swelling

CHAPTER 5: DISCUSSION

Muscle Thickness

Isometric Strength

Muscle Swelling

Total Body Water

Limitations

29

Conclusions

Appendix A: Exclusionary Criteria Checklist 37

Appendix B: Physical Activity Readiness Questionnaire 


\section{List of Tables}

Table 1: Demographics $\quad 26$

Table 2: Total Body Water $\quad 26$

$\begin{array}{ll}\text { Table 3: Isometric Strength } & 27\end{array}$

$\begin{array}{ll}\text { Table 4: Muscle Thickness } & 28\end{array}$

Table 5: Acute Changes in Muscle Thickness 29 


\section{List of Figures}

Figure 1: Total Body Water Across Menstrual Cycle

Figure 2: Isometric Strength Across Menstrual Cycle

Figure 3: Muscle Thickness Across Menstrual Cycle

Figure 4: Acute Changes in Muscle Thickness Across Menstrual

Cycle 


\begin{abstract}
Women are often excluded from research studies interested in changes in muscle size and strength due to confounding influence that the menstrual cycle may have on these variables. Although there is considerable data showing that strength may fluctuate throughout the menstrual cycle, the thesis that muscle size changes throughout the cycle is based on the premise that changes in body water throughout the menstrual cycle may influence the size of the muscle. Despite this suggestion there is no experimental data demonstrating that the menstrual cycle has any influence on muscle size. Purpose: The purpose of this study is to examine the changes in muscle size and strength throughout the menstrual cycles in females and to compare these values to those to a control group of time-matched males. Methods: 12 males and 16 females visited the laboratory on four separate occasions. The first visit consisted of paperwork, familiarization with cycle tracking and isometric strength assessment. During the second visit, which was 1-7 days after the first day of the menstrual phase, measurements of muscle thickness, isometric strength, and body water were recorded. The third visit took place during the ovulation phase. This phase typically occurs between 6-10 days after menstruation, ovulation was predicted through the Flo App. Visit four took place during the luteal phase which typically occurs between days 22-25 of the menstrual cycle. Males scheduled their second visit 1-7 days following visit 1 . This visit represented the "menstrual phase" for male participants. Hereafter, male participants followed a mock menstrual cycle via the Flo app. During all visits, muscle thickness, isometric strength of the biceps and body water composition were measured. In addition to this, the participants were asked to complete 4 sets of biceps curls at $70 \%$ of $1 \mathrm{RM}$ to
\end{abstract}


volitional failure, in one arm during visits 2,3 and 4 . Additional measures of muscle thickness were taken following exercise. All participants were instructed to continue normal eating and water consumption habits during this study. A food log was used to ensure that the same food and drink are consumed 24 hours prior to each visit. Results: For muscle thickness there was no interaction $(p=0.73)$. In addition, there was no main effect for time $(p=0.93)$. However, there was a main effect for sex. Males had higher muscle thickness values at all time points compared to females $(\mathrm{p}<0.001)$. For acute changes in muscle thickness (swelling)there was no interaction $(p=0.28)$. In addition, there was no main effect for time $(p=0.12)$. However, there was a main effect for sex. Males had higher acute changes in muscle thickness all time points compared to females $(\mathrm{p}<0.001)$. For total body water there was no interaction $(\mathrm{p}=0.66)$. In addition, there was no main effect for time $(\mathrm{p}=0.97)$. However, there was a main effect for $\operatorname{sex}(\mathrm{p}<0.001)$. Males had greater total body water at all time points compared to females. For isometric strength there was no interaction $(p=0.23)$. In addition, there was no main effect for time $(p=$ 0.73). However, there was a main effect for sex. Males had higher isometric strength values at all time points compared to females $(\mathrm{p}<0.001)$. Conclusions: Phase of the menstrual cycle does not appear to influence muscle thickness, isometric strength or total body water. 


\section{Chapter 1: Introduction}

\section{Rationale}

Changes in muscle size resulting from resistance training are often measured using b-mode ultrasound (1-3). This technique allows accurate assessment of skeletal muscle size, and has been shown to track similarly to more sophisticated techniques such as magnetic resonance imaging and computed tomography (4). One limitation of muscle imaging techniques is an inability to differentiate between changes in skeletal muscle size due to hypertrophy, and changes in skeletal muscle size due to swelling, or Edema. Recently, Damas et al. (5) has suggested that skeletal muscle size may not be measureable until 12-weeks into a resistance training program and that early changes in muscle size are likely attributed to swelling/edema. This suggestion is based largely on changes in echo-intensity of ultrasound images (i.e., the color of the ultrasound pixels) (5). Interestingly, Yitzchaki et al. (6) demonstrated that echo-intensity cannot accurately detect the presence of acute muscle swelling. Thus, when trying to minimize the potential influence of swelling, the best approach may be through study design (i.e., timing of measurements). For example, Buckner et al. (7) has demonstrated that muscle swelling does not accumulate over the course of a training week and appears to have little influence beyond what happens following the initial exposure to resistance exercise. Buckner et al. (7) suggests that the acute swelling response itself can be used as a means to detect the presence of muscle swelling. Meaning, if a muscle is able to swell, this provides some evidence that the muscle wasn't swollen to begin 
with. Despite meaningful work into better understanding the role that swelling may play in the context of detecting skeletal muscle growth, some researchers exclude women from their studies under the premise that changes in body water throughout the menstrual cycle may influence the size of the muscle. Despite their exclusion, it is not currently known if the menstrual cycle has any influence on changes in muscle thickness/size.

The thesis that the menstrual cycle may influence different physiological variables is largely related to changes in hormones. It has been previously demonstrated that during the follicular phase of the menstrual cycle, oestrogen levels rise to a peak and then fall a day or two before ovulation, while during the luteal phase the oestrogen levels remain fairly stable (1). Interestingly, progesterone levels are negligible during the follicular phase, rising to a peak during the luteal phase (1). Based on the hypothesis that oestrogen can lead to increases muscle strength, it has been suggested that strength may peak in the follicular phase of the menstrual cycle. This is supported by the findings of Phillips et al. (9) who examined isometric strength in the adductor pollicis throughout the menstrual cycle, finding that strength peaked around the time of ovulation (coincided with peak oestrogen levels). Similarly Sarwar et al. (10) observed an $11 \%$ increase in quadriceps and hand grip strength during the mid-cycle compared to the follicular and luteal phases. Authors also attribute this increase to changes in oestrogen (10). Despite many studies examining changes in muscle strength throughout the menstrual cycle, there is far less work examining changes at the tissue level. Notably, no study has examined change in muscle thickness throughout the different phases of the menstrual cycle. However, it has been demonstrated that bodyweight does change throughout the cycle, with weight peaking around the late luteal phase (11). Such data has been influential in the skeletal muscle literature 
as it is common to exclude women from research studies examining changes in muscle size based on the premise that changes in hydration status throughout the menstrual cycle may influence the size of the muscle $(10,12)$. Thus, an increase in water retention may be mistaken for growth or edema. However, it has never been experimentally demonstrated that changes in water content are reflected at the muscle level. The purpose of this study is to examine the changes in muscle size, isometric strength and body water throughout the menstrual cycles in females and to compare these values to those of a control group of time-matched males.

\section{Problem Statement}

There has been a very limited involvement of women as participants in exercise science research. The biggest argument for excluding women has been that it is difficult to account for the changes in muscle strength, specifically, throughout the menstrual cycle $(9,10)$. This has also been extended to muscle size. Despite a tendency to exclude women from studies examining changes in muscle size $(5,7)$, no current study has examined changes in muscle size throughout the menstrual cycle. Changes in muscle size have been inferred from the literature examining fluctuations in body weight (11). This present study could help facilitate more research in female populations, particularly if no changes in muscle thickness are observed throughout the mensural cycle. Results of the present study may allow researchers to expand their recruitment pool, increasing the generalizability of research findings to a larger portion of the population. Most importantly, results of the present study will help to determine how much (if any) influence the menstrual cycle has on muscle thickness. 


\section{Research Variables}

Independent: 1) sex: male or female

2) normal menstrual cycle (28-32 days)

Dependent: 1) isometric strength

2) body water composition

3) muscle thickness

\section{Hypotheses}

Hypothesis 1: Compared to males, females will not have changes in isometric strength throughout the menstrual cycle.

Hypothesis 2: Compared to males, females will not have changes in body water composition throughout the menstrual cycle.

Hypothesis 3: Compared to males, females will not have changes in muscle thickness measurements throughout the menstrual cycle.

Hypothesis 4: Compared to males, females will not have changes in muscle swelling values throughout the menstrual cycle.

\section{Operational Definitions}

- College Age: 18-35 
- One Repetition Maximum: the most one individual can lift one time with good form

- Ovulation: the release of an egg during the menstrual cycle

- Anovulation: the body does not release an egg during the menstrual cycle

- Body Water Composition: percentage of body composition pertaining specifically to total body water

- Muscle Thickness: An estimate of muscle size, derived through a one-dimensional B-mode ultrasound image. MTH is measured as the distance from the muscle-bone interface to the muscle-fat interface

- Isometric Strength Testing: The peak torque produced by a muscle as it contracts while pulling against an immovable object

- Menstrual Cycle: regular cycle consisting of 28-32 days with ovulation occurring

- Muscle Swelling: An acute increase in muscle thickness as measured through Bmode ultrasound, expressed as the change in muscle thickness from before to after an exercise bout

\section{Assumptions}

1. The researcher assumes participants will provide honest and accurate responses to all questions.

2. The researcher assumes all participants will adhere to all instructions and will give required time and effort during all visits.

3. Participants will give a maximal effort during all muscular strength testing and training sessions. 


\section{Limitations}

1. The sample size and demographics such as age and having a normal menstrual cycle may limit the generalizability of the results for other age groups or populations.

2. The exclusion of women taking hormonal birth control will be a limitation.

3. Not testing the levels of hormones throughout the different phases of the menstrual cycle may be a limitation.

4. The accuracy of predicting ovulation based off of the Flo Tracking App, and with the ovulation test kits may be a limitation.

5. Participants will not be allowed to continue the study if they have any known reproductive and/or endocrine disorder.

6. Participants may not be familiar with strength testing protocols or exercise protocols used for this study, this will be addressed through the familiarization trial as well as before each experimental trial begins.

\section{Delimitations}

1. The researcher chose the age group due to the study being conducted on a college campus, and to be able to recruit a larger sample.

2. Participants will be asked to avoid any exercise 24 hours prior to each exercise visit to avoid fatigue, delayed onset muscle soreness or dehydration. 


\section{Significance}

The goal of this research is to look at the changes in isometric strength, body water composition, and muscle thickness during the menstrual cycle to implicate the inclusion of women as participants in exercise science research. Although women make up $50.8 \%$ of the population, their representation in the exercise science literature is lacking. In many cases, their exclusion is due to the confounding influence that the menstrual cycle may have on various measures. Of interest in the particular study are changes in muscle size and muscle swelling. A recent study by Buckner et al. (7) examined changes in muscle size in the early phases of resistance training. Authors were interested in detecting how early skeletal muscle growth might occur. However, women were excluded, based on the hypothesis that changes in fluid retention throughout the menstrual cycle may influence muscle thickness compromising the ability to detect skeletal muscle growth. Results from this study will allow future researchers to have larger sample sizes, compare men to women rationally, as well as inform researchers on how to account for the menstrual cycle if any changes do present themselves. 


\section{Chapter 2: Review of the Literature}

\section{Introduction}

The purpose of this literature review is to determine what changes have been seen in strength and body water composition in women during the menstrual cycle. It will also show what is missing from the current literature, specifically in this case, muscle thickness measurements. It is organized by presenting what the current literature reflects today. This review will go through different hormone levels at different phases in the menstrual cycle, how these hormone levels affect strength and overall body water, and the difference between women taking hormonal contraceptives versus not taking contraceptives.

\section{Background}

Historically, within the field of exercise science, women have been largely excluded from research studies. For example, the well-known equation to predict maximal heart rate (220 - age) was calculated from a sample of North American and European males (13). Perhaps more famously, the calculation of a metabolic equivalent (1 MET) comes from a study in 1890 that consisted of a sample of one 40 year old male with a body mass of $\sim 70 \mathrm{~kg}$ (14). In more modern time, a pattern of excluding women is still apparent. For example, a review on the blood flow restriction research found that out of 4,335 participants included in blood flow restriction studies only $29 \%$ and $17 \%$ of the total sample included women for acute and chronic training studies 
respectively (15). When looking for a rationale behind exclusion, the influence of the menstrual cycle on outcomes of interest if often cited. For example, Sakamaki et al. (16) observed muscle growth in both the follicular and the luteal phases of the menstrual cycle, finding that the greatest increase in muscle size was observed during the luteal phase. Methodologically, it is believed that the menstrual cycle may result in fluid retention in muscle. If true, this would confound the results in studies trying to induce skeletal muscle hypertrophy. This is most obvious when examining studies trying to elucidate the time-course of skeletal muscle growth. Damas et al. (8) examined early changes in skeletal muscle growth, excluding women due to confounding influence that the menstrual cycle may have on edema. Similarly, Buckner et al. (7) examined contributions of growth and swelling during the first week of resistance training. Authors excluded women due to the influence that the menstrual cycle may have on swelling/edema.

\section{Menstrual Cycle and Strength}

It is hypothesized that the menstrual cycle has an effect on strength, body weight, and body water in women. The inability to account for these fluctuations are the reason why many times women are excluded from exercise science research. It is proposed that the fluctuations occur due to changes in hormone levels, specifically estrogen, estradiol, and progesterone. A study done by Phillips et al. (9) examined the effect of estrogen and progesterone on skeletal muscle strength during the menstrual phase. They found that significant changes occurred in muscle strength during the follicular phase when compared to the luteal phase, as well as a rapid decrease in strength around ovulation. Although, due to limitations with tracking hormone levels, they did not find any correlation between hormone serum concentrations and strength decrements. Sarwar et al. (10) examined muscular strength, fatigability and contractile rate amongst women on oral 
contraceptives (OC) and those not taking birth control. They found no changes over time for any variable for the women taking birth control, although there were increases in the quadriceps strength, relaxation rate, and fatigue rate for women not taking birth control when compared to those on OC. A study done by Janse de Jonge (17), looked at the difference in isokinetic quadriceps strength as well as isometric handgrip strength during the follicular, menstrual and luteal phases of the menstrual cycle in women who were not taking oral contraceptives and had regular periods ranging from 28-32 days. They also found no correlations between any of the strength parameters and the serum concentrations of oestrogen, progesterone, lutenizing hormone and follicular stimulating hormone. Lebrun et al. (18), also showed no differences in isokinetic strength of the knee extensors between the follicular and luteal phases. Several other studies have found no changes in skeletal muscle strength over the menstrual cycle (19-22). The main problem in the measurement of maximum voluntary strength is ensuring that the contraction truly reflects the maximum force-generating capacity of the muscle (23). Jonge et al. (17) reported no significant changes over the phases of the menstrual cycle for isometric quadriceps strength with superimposed electrical stimulation to account for maximal neural activation during maximal voluntary contraction. They also reported no changes over the menstrual cycle for isokinetic strength and fatigability of the knee flexors and extensors, as well as no significant changes in handgrip strength throughout the cycle. In addition, no significant changes in the extent of neural activation were found throughout the menstrual cycle (17).

Although changes in strength are inconsistent throughout the menstrual cycle, it is important to consider that studies that have included both women taking birth control and women not on birth control have observed changes only in the groups that were not on birth control (10). Women 
who are not on oral contraceptives or hormonal birth control experience typical fluctuations in hormone levels throughout the cycle, whereas women who are taking oral contraceptives and hormonal birth control have a controlled amount of hormone introduced to their body for the entirety of their menstrual cycle. These levels of hormones are consistent throughout multiple periods, if not for the entire time an individual is taking the contraceptive. Oral contraceptives appear to decrease or eliminate the influence of the menstrual cycle on strength outcomes (10). The early formulas for birth control contained much higher dosages of both estrogens and progestins, which may have had a greater influence on strength outcomes due to superphysiological levels of hormones. However, since the androgenic actions of the progesterone component in newer preparations is smaller, there is likely no significant influence of the exogenous hormones on strength (18).

\section{Menstrual Cycle and Muscle Size}

Despite considerable work on performance measures throughout the menstrual cycle, far less work exists examining changes in skeletal muscle morphology. In fact, assumptions regarding the menstrual cycle's influence on skeletal muscle size permeates from the literature which has demonstrated changes in body weight throughout the cycle. Many women complain of a feeling of weight gain in the premenstrual phase but there is evidence that regular physical activity can alleviate some of the premenstrual symptoms, including fluid retention and cramps (24). Women who are not on oral contraceptives or hormonal birth control experience these fluctuations in hormone levels throughout the cycle, whereas symptoms may be alleviated in the presence of birth control. Although many studies have found that bodyweight does not change throughout the cycle $(19,25,26)$, when multiple (daily) time points were included Watson and Robinson (11) 
noted a peak in weight during the late luteal phase. However, a study done by Lebrun et al, reported no changes in body weight, percent body fat, or sum of skinfold measurements in women during the follicular phase and the luteal phase of the menstrual cycle (19). Thus, there are discrepant findings as to whether oestrogen and progesterone effect fluid retention. It has been alternatively hypothesized that fluid distribution as opposed to total fluid retention may change throughout the menstrual cycle (27). If this is true, these changes in fluid retention and/or fluid distribution could potentially influence muscle size and acute swelling measurements.

\section{Muscle Thickness}

Brightness mode ultrasound (B-mode) is used to examine acute changes in muscle thickness as shown by Buckner et al. 2017 (7). B-mode ultrasound is a highly reliable method for measuring muscle thickness in both males and females (28) and has been shown to produce measurements similar to those of more widely known techniques (such as magnetic resonance imaging or computed tomography) (4). Although b-mode ultrasound cannot differentiate the presence of swelling or fluid accumulation within a muscle (6), inducing swelling through exercise may provide valuable information on the baseline state of the muscle (7).

\section{Muscle Swelling}

Cell swelling is a hypothesized mechanism for skeletal muscle growth. Haussinger (29, 30) suggests that acute swelling may act as an anabolic signal resulting in a shift towards anabolism. It has been suggested that amino acids are taken up into cells by sodium-ion dependent transport systems, converting an electrochemical gradient into an osmotically active amino acid gradient, which ultimately causes a fluid shift of water into the cell (29). We believe the potential fluid 
retention during the menstrual cycle may affect this shift of water into the cell during swelling, therefore causing an exaggerated swelling response. Although cell swelling is only a hypothesized mechanism of skeletal muscle growth, it is a repeatable phenomenon which has been examined across a variety of resistance training protocols and is believed to play a role in the anabolic process observed with resistance exercise $(2,31)$. From a study design perspective, many studies will examine the acute muscle swelling response as an indirect marker of the anabolic potential of a resistance training stimulus $(7,31,32)$. If acute swelling changes throughout the menstrual cycle, in may play a confounding role in the ability to draw relevant conclusions from the acute swelling response to exercise.

To provide some support for the cell swelling hypothesis, Yasuda et al. (33) observed that concentric exercise combined with BFR resulted in both a greater acute muscle swelling response and greater increase in muscle size over a 6-week period compared to a group performing eccentric exercise in combination with BFR. Thus, authors make the suggestion that the larger degree of swelling may explain the larger degree of growth. Although it is still not known if swelling is necessary to induce skeletal muscle growth, it seems that all known training protocols that produce growth also produce some level of swelling. This is true for high load (2), low load, low load with BFR $(2,31)$ and NO-LOAD (2) exercise. In addition, Buckner et al. (7) found that a muscle swells to a similar degree when repeatedly exposed to the same exercise stimulus over a training week. Authors suggest that this may provide important information regarding the effectiveness of the exercise bouts. 


\section{Chapter 3: Methodology}

\section{Participants}

Fifteen females between the ages of 18-35 who are not currently taking oral contraceptives were recruited for this study. In addition, 15 men between the ages of 18-35 were also be recruited and served as a time matched control sample.

\section{Study Inclusion/Exclusion Criteria}

\begin{tabular}{|c|c|}
\hline $\begin{array}{l}\text { Inclusion Criteria (Intervention and Control } \\
\text { Group) }\end{array}$ & $\begin{array}{l}\text { Exclusion Criteria (Intervention and Control } \\
\text { Group) }\end{array}$ \\
\hline - $\quad$ Between the ages of $18-35$ & - $\quad$ Taking hypertensive medications \\
\hline $\begin{array}{l}\text { - No Orthopedic problems preventing } \\
\text { strength testing }\end{array}$ & \\
\hline - Non-smokers & $\begin{array}{l}\text { - } \text { Irregular menstrual cycle (including } \\
\text { amenorrhea, or missed periods) }\end{array}$ \\
\hline - $\quad$ Not on hypertensive medications & $\begin{array}{l}\text { - Known reproductive and/or endocrine } \\
\text { disorder }\end{array}$ \\
\hline $\begin{array}{l}\text { - Not taking hormonal contraceptives for at } \\
\text { least } 6 \text { months }\end{array}$ & - Pregnant or planning to become pregnant. \\
\hline 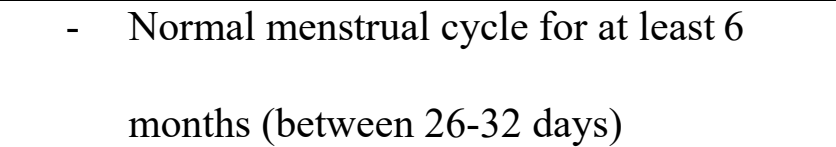 & \\
\hline
\end{tabular}




\section{Study Overview}

Participants visited the laboratory on four separate occasions. The first visit consisted of paperwork, familiarization with the Flo app for cycle tracking, isometric strength assessment, muscle thickness and body water. During the second visit, which was 1-7 days after the first day of the menstrual phase, which is defined by the onset of bleeding, measurements of muscle thickness, isometric strength, and body water were recorded. The third visit was during the ovulation phase. This phase occurs typically between 6-10 days after menstruation, ovulation was predicted through the Flo App, as well as, validated with at home ovulation kits (MomMed Manufacturers), provided by the study staff during visit one (measured via urine sample). Visit four took place during the luteal phase which typically occurs between days 22-25 after the onset of bleeding during the cycle. Males scheduled their second visit 1-7 days following visit 1 . This visit represented the "menstrual phase" for male participants. Hereafter, male participants followed a mock menstrual cycle via the flow app. During all visits, muscle thickness, isometric strength of the biceps and body water composition was measured in both arms. In addition to this, the participants were asked to complete 4 sets of biceps curls at $70 \%$ of 1RM to volitional failure, in one arm for visits 2, 3 and 4. Sixty second rest periods were had between sets, and muscle thickness measurements were taken immediately (under 30 seconds) upon conclusion of the 4th exercise set. All participants were instructed to continue normal eating and water consumption habits during this study.

\section{Measurements}

Height/Body Mass

Height and body mass were measured using a standard scale and stadiometer. 


\section{One Repetition Maximum}

Each individual's strength was tested on their randomized experimental arm, this was measured using a one-repetition maximum (1-RM; the heaviest an individual can lift one time with good form). A 10-repetition light warm up and a 3-repetition, slightly heavier, warm up both took place before testing commenced. Participants were supervised by trained personnel during all strength testing. This measurement was taken during the first visit.

\section{Muscle Thickness}

Ultrasound measurements of muscle size were taken on the front of the upper arm. The probe was coated with gel and held lightly against the skin. Measurements were taken at 70\% the distance from the acromion process to the lateral epicondyle. All measurements were taken by the same trained technician.

\section{Isometric Strength}

For upper body strength testing, participants were asked to flex their arm against an immovable object as hard as possible to determine their isometric strength. Two contractions per arm with each lasting approximately 3 seconds.

\section{Body Water Composition}

Body water composition was measured using the InBody® 570 BodyComposition Analyzer (Biospace, Inc. Seoul, Korea). The InBody uses bioelectrical impedance technology to estimate 
total body water. After cleaning their hands and feet with an alcohol wipe, the participants were then told to step on the InBody with heels in contact with the electrodes. When weight measurement was completed, the participant then held the handles, with thumbs in contact with the electrodes and arms extended at 20 degrees of shoulder abduction while the test took place making sure to not touch the trunk of the body. The participant was also instructed to remain still and silent during the assessment.

\section{Tracking Protocol}

Participants were asked to track their cycle as well as symptoms on the Flo app, this portion of the protocol took approximately 2 minutes daily to achieve. The participant was then asked to forward this data to the PI upon the completion of the month via email. The Flo app is a cycle tracker that predicts phase of the cycle based on the onset of menstruation, which is defined as the first day of bleeding. Along with tracking their cycle on the app, female participants received an at home ovulation test kit (MomMed) during the initial visit to the lab. Female participants were asked to test their ovulation on the day that the app predicted ovulation and the day before, the at home ovulation kits tested luteinizing hormone via urine sample. Written instructions were provided along with the kit and the Co-PI reviewed these procedures with the participant. The participant then forwarded these results to the PI when they visited the lab for visit 2.

\section{Equipment}

The Inbody Bioelectrical Impedance (South Korea) machine was used to determine body water composition throughout the study. MomMed at home ovulation test kits were given to each participant during their visit one to the lab, these kits included detailed instructions on how 
to test for ovulation provided by MomMed, any questions were referred to the PI and study staff regarding the kits. Isometric Strength was tested using a handgrip dynamometer located in the USF Muscle Laboratory. One repetition max was measured using free-weight dumbbells under supervision of study staff.

\section{Procedures}

- Visit 1

Consent Form and Questionnaires

Informed consent was obtained prior to completion of all questionnaires and any testing. Participants were then asked to complete and go through the exclusionary criteria (Appendix A) questionnaire and the physical activity readiness questionnaire (Appendix B). If they qualified for this study after completing these forms, their height and weight were measured. Following height and weight, participants were then familiarized with how to track symptoms and cycle changes on the Flo app, participants were also familiarized with the isometric strength measurement. During this visit each participant completed a one repetition max (1RM) test to determine their exercise weight for the other visits. Lastly, during this visit participants were given at home ovulation kits along with instructions of when to take the test and how to report results to the PI.

\section{- Visit 2}

After visit 1, the participants were instructed to contact the PI on the first day of their menstrual cycle, this was recorded by the first bleeding day (menstrual phase). The participant came into the lab between day 1 and day 7 of the menstruation phase. When 
participants visited the lab for visit 2, total body water was measured using the InBody Bioelectrical Impedance (South Korea, 1996). After body water was measured, values of muscle thickness in both arms using B-mode ultrasound were taken. In addition, participants complete an isometric strength assessment in both arms prior to exercise. The participants then completed 4 sets of biceps curls at 70\% 1RM to volitional failure in a randomized arm, this remained the exercising arm throughout the duration of the study, muscle thickness measurements were taken again immediately (within 30 seconds) postexercise. Participants did not have to be fasted for this study, instead, participants were asked to complete a diet $\log 24$ hours prior to their second visit to the lab. We then made two copies of the diet log. One was kept in the lab for our records and the other was given back to the participant. The participant was then instructed to replicate this diet before visits 3 and 4. All measurements during visits 3 and 4 were taken in an identical sequence to that of visit 2 .

\section{- Visit 3}

On visit 3 participants came to the lab and upon arrival measurements of muscle thickness in both arms, isometric strength, and body water composition were taken. Participants then completed 4 sets of biceps curls to volitional failure at $70 \% 1 \mathrm{RM}$, muscle thickness was taken pre- and post-exercise. There were 60 second rest periods between exercise sets. Muscle thickness measurements were immediately taken within 30 seconds upon the conclusion of exercise. This visit took place during ovulation which was determined by the Flo App cycle calendar and validated by the at home ovulation kit to determine luteinizing hormone. 


\section{- Visit 4}

Visit 4 took place between days 22-25 after the onset of the cycle, defined by the first day of bleeding, according to the Flo App tracker, this is referred to as the luteal phase. This visit consisted of muscle thickness, isometric strength, and body water composition measurements. Participants then completed one last exercise bout of 4 sets of biceps curls to volitional failure at $70 \% 1 \mathrm{RM}$ with 60 second rest periods between sets. Muscle thickness measurements were also taken pre- and post- exercise.

\section{- Familiarization Protocol}

Participants were familiarized with both the Flo tracking app, and isometric strength measurement which was specific to the biceps curl model.

\section{Statistical Analysis}

Using the SPSS 24.0 statistical software package (SPSS Inc., Chicago, IL), a 2x4 (group x time) repeated measure ANOVA was used to determine any differences in muscle thickness and torque between sexes in the upper body. If there were interactions, we ran one-way ANOVAs across time within each sex, as well as across sex within each time point. If no interactions were detected, we looked at main effects of time and sex. Statistical significance for all tests were set at an alpha level of 0.05 . 


\section{Chapter 4: Results}

\section{Demographic}

Descriptive data is presented as mean (SD) and displayed in table 1. A total of 28 women $(n=16)$ and men $(n=12)$ completed the study.

Table 1: Demographics

\begin{tabular}{cccc} 
& Men $(\mathbf{n}=\mathbf{1 2})$ & Women $(\mathbf{n}=\mathbf{1 6})$ & $\boldsymbol{P}$-value \\
\hline Height (cm) & $179.0(11.2)$ & $161.2(6.2)$ & $<\mathbf{0 . 0 0 1}$ \\
Body Mass (kg) & $80.3(8.1)$ & $63.4(11.7)$ & $<\mathbf{0 . 0 0 1}$ \\
Age (yrs) & $24(4)$ & $23(2)$ & $\mathbf{0 . 4 7}$ \\
1RM (kg) & $25.4(4.9)$ & $12.2(3.2)$ & $<\mathbf{0 . 0 0 1}$ \\
\hline
\end{tabular}

Data is displayed as means (SD).

$1 \mathrm{RM}=$ one repetition maximum strength

\section{Total Body Water}

For total body water there was no sex $(x)$ time interaction $(p=0.66)$. In addition, there was no main effect for time $(p=0.97)$. However, there was a main effect for $\operatorname{sex}(p<0.001)$.

Males had greater total body water at all time points compared to females.

Table 2: Total Body Water (kg)

\begin{tabular}{lll} 
& Men $(\mathbf{n}=\mathbf{1 2})$ & Women $(\mathbf{n}=\mathbf{1 6})$ \\
\hline Menstrual & $49.6(6.6)$ & $32.3(4)$ \\
Ovulation & $49.8(7)$ & $32.4(4)$ \\
Luteal & $49.5(7)$ & $32.3(3.9$ \\
\hline
\end{tabular}


Total Body Water

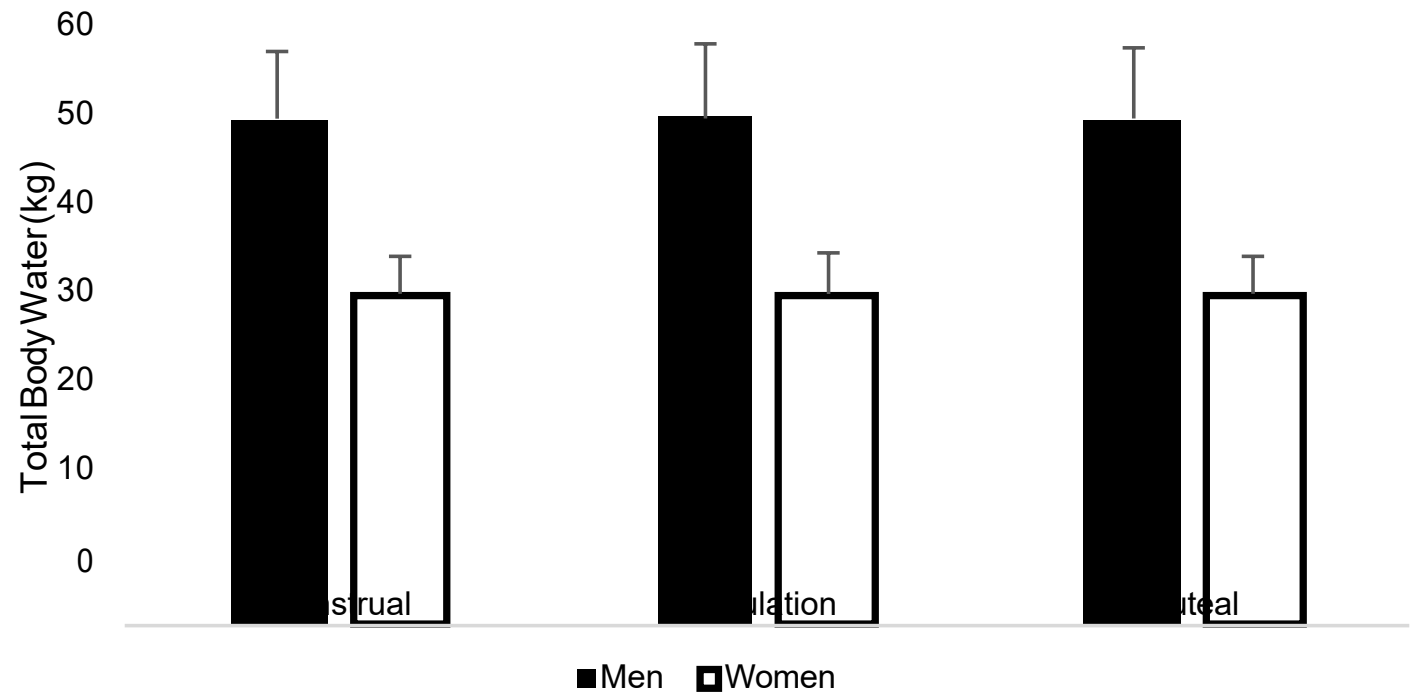

Figure 1: Total Body Water Across Menstrual Cycle. Displays total body water values across the menstrual cycle for both men and women.

\section{Isometric Strength}

For isometric strength there was no $\operatorname{sex}(\mathrm{x})$ time interaction $(\mathrm{p}=0.23)$. In addition, there was no main effect for time $(p=0.73)$. However, there was a main effect for sex. Males had higher isometric strength values at all time points compared to females $(\mathrm{p}<0.001)$.

Table 3: Isometric Strength (newtons)

\begin{tabular}{lll} 
& Men $(\mathbf{n}=\mathbf{1 2})$ & Women $(\mathbf{n}=\mathbf{1 6})$ \\
\hline Menstrual & $290.05(44.36)$ & $154.59(37.86)$ \\
Ovulation & $278.44(45.77)$ & $153.69(35.40)$ \\
Luteal & $286.54(37.79)$ & $160.88(37.48)$ \\
\hline
\end{tabular}




\section{Isometric Strength}

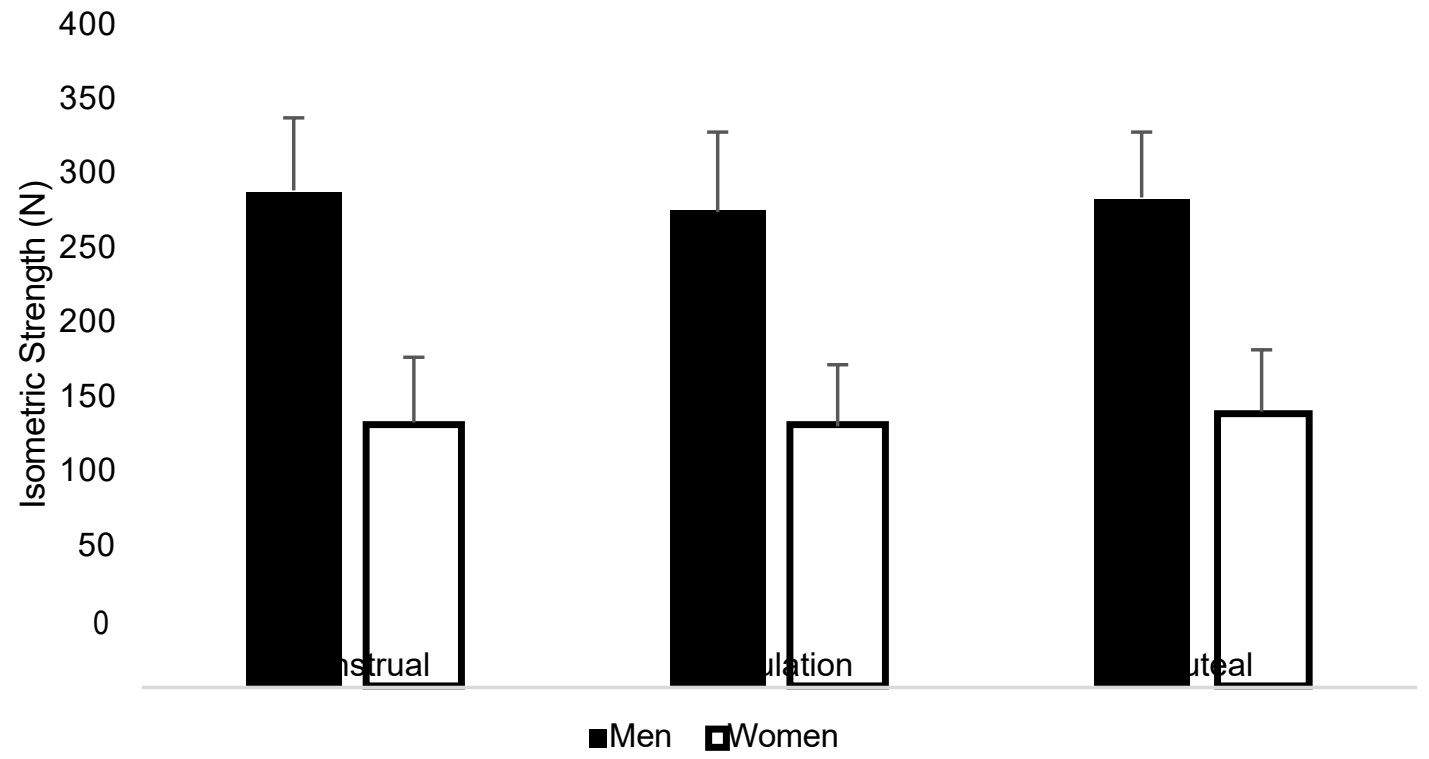

Figure 2: Isometric Strength Across Menstrual Cycle. Displays isometric strength values across the menstrual cycle for both men and women Muscle Thickness

For muscle thickness there was no sex $(\mathrm{x})$ time interaction $(\mathrm{p}=0.73)$. In addition, there was no main effect for time $(\mathrm{p}=0.93)$. However, there was a main effect for sex. Males had higher muscle thickness values at all time points compared to females $(\mathrm{p}<0.001)$.

Table 4: Muscle Thickness (cm)

\begin{tabular}{lll} 
& Men $(\mathbf{n}=\mathbf{1 2})$ & Women $(\mathbf{n}=\mathbf{1 6})$ \\
\hline Menstrual & $4.06(0.67)$ & $2.74(0.39)$ \\
Ovulation & $4.08(0.67)$ & $2.73(0.44)$ \\
Luteal & $4.08(0.68)$ & $2.72(0.45)$ \\
\hline
\end{tabular}




\section{Muscle Thickness}

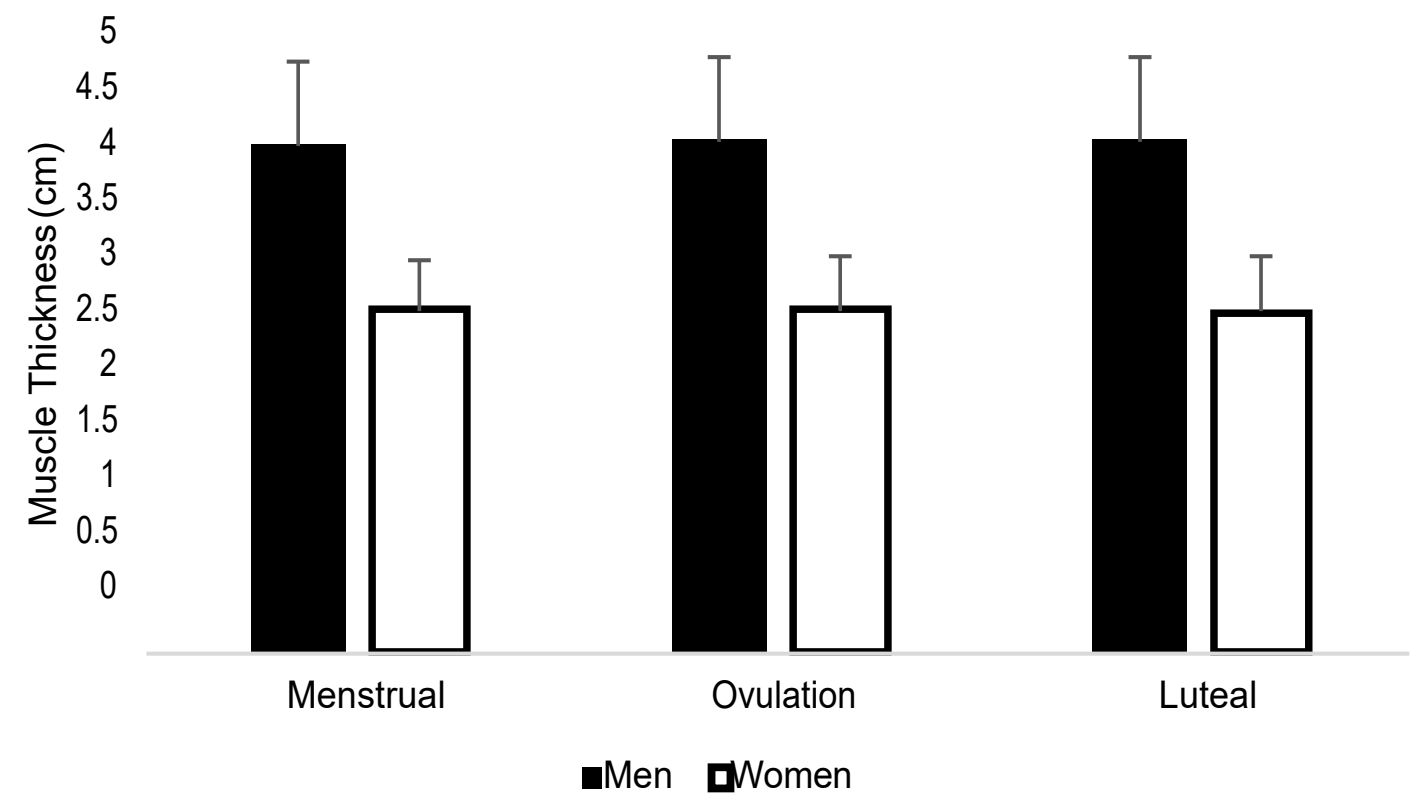

Figure 3: Muscle Thickness Across Menstrual Cycle. Displays muscle thickness values across the menstrual cycle for both men and women Muscle Swelling

For acute changes in muscle thickness (swelling) there was no sex (x) time interaction $(\mathrm{p}=$ 0.28). In addition, there was no main effect for time $(\mathrm{p}=0.12)$. However, there was a main effect for sex. Males had higher acute changes in muscle thickness all time points compared to females $(\mathrm{p}=0.001)$

Table 5: Acute Changes in Muscle Thickness (cm)

\begin{tabular}{lll} 
& Men $(\mathbf{n}=\mathbf{1 2})$ & Women $(\mathbf{n}=\mathbf{1 6})$ \\
\hline Menstrual & $0.58(0.10)$ & $0.40(0.11)$ \\
Ovulation & $0.50(0.10)$ & $0.41(0.11)$ \\
Luteal & $0.43(0.14)$ & $0.37(0.10)$ \\
\hline
\end{tabular}




\section{Muscle Swelling}

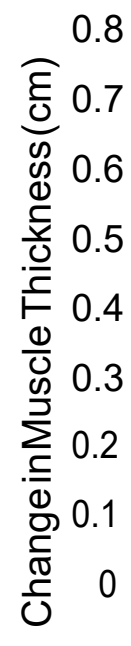
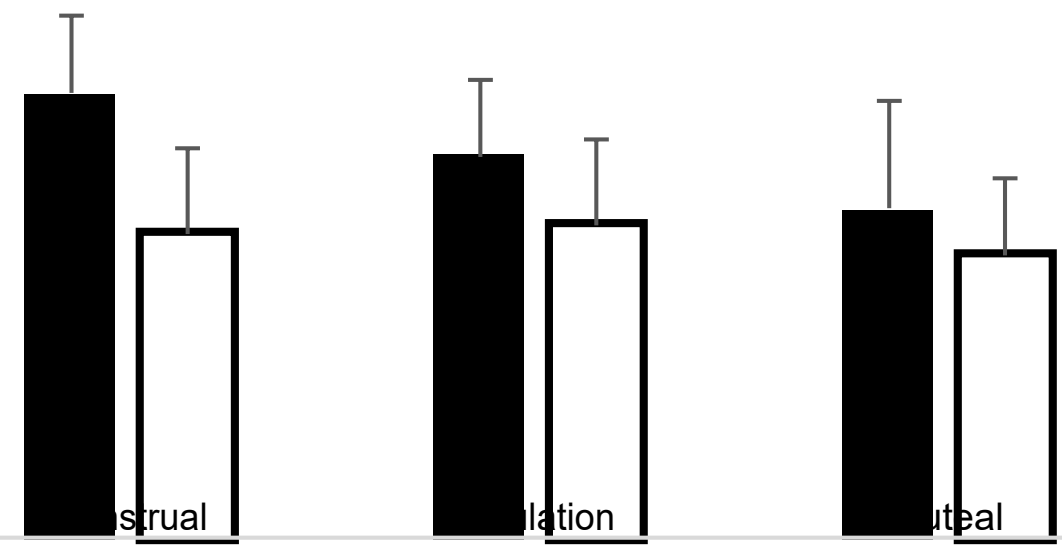

-Men $\mathbf{D W o m e n}$

Figure 4: Acute Changes in Muscle Thickness Across Menstrual Cycle . Displays values for acute changes in muscle thickness across the menstrual cycle for both men and women. 


\section{Chapter 5: Discussion}

The purpose of this study was to examine changes in muscle thickness, isometric strength, and body water composition throughout the menstrual cycle and to determine if there were differences across time between men and women. Results demonstrated that there were no changes for any of the measurements taken across time. In addition, there were no sex differences in any variable across time. However, there were main effects for sex in all measurements, with men overall having greater overall values for isometric strength, muscle thickness, total body water and acute muscle swelling.

\section{Muscle thickness}

When measuring skeletal muscle growth using b-mode ultrasound, it is often difficult to differentiate between real growth and swelling/edema (41). Buckner et al (7), examined changes in muscle thickness throughout a single training week, suggesting that swelling does not accumulate across time. Although it has been suggested that imaging procedures, such as echointensity, may be able to inform us on the presence of swelling (5), recent work has suggested that this may to provide valuable information on the swelling state of a muscle (6). As a result, the best way to account for confounding factors may be through study design. The results of our study suggest that the menstrual does not seem to influence muscle thickness. Therefore, this may not be an important consideration when studying muscle adaptations across time. The results from this study suggest that the exclusion of women from studies examining changes in 
muscle thickness due to influence of the menstrual cycle on muscle thickness measurements may not be necessary.

\section{Muscle Strength}

The present study observed no influence of the menstrual cycle on isometric strength. Similarly, Arazi et al (33) showed no significant difference between muscular strength and endurance in three phases of the menstrual cycle. Authors observed no significant changes in anaerobic power during all three phases of the menstrual cycle, however there were differences in luteinizing hormone and follicular stimulating hormone levels in the three different phases (35). Ekenros et al (36), found no significant difference in muscle strength between women taking oral contraceptives (OC) and women not taking oral contraceptives (non-OC). However, they did identify a change in knee extensor maximal isokinetic muscle strength in the OC cycle between the early follicular phases and the luteal phase (36). A study done by DiBrezzo (20) and colleagues also found similar results to those of Ekenros (36) and Arazi (33). Authors observed that the different phases of the menstrual cycle had no significant effect upon the relationships between knee extension and flexion strength, body weight, percent of body fat and endurance (20). A study by Giacomoni et al. (37), examined anaerobic performance during different menstrual cycle phases, finding no significant differences in maximal anaerobic performance across the phases of the cycle. Authors did however suggest that premenstrual or menstrual syndrome symptoms may affect the outcomes of anaerobic performance possibly through the stretch-shortening cycle (37). Giacomoni et al. (37), also showed no significant differences between women taking monophasic oral contraceptives and women not taking oral 
contraceptives even with the presence of increase progesterone between the follicular and luteal phase in women with ovulatory cycles (non-OC group).

\section{Muscle Swelling}

It has been suggested that acute muscle swelling may act as an anabolic signal resulting in a shift towards anabolism (i.e., muscle growth) (29). Thus, even if muscle thickness doesn't change over time, is there are changes in swelling, it may provide important information on the anabolic potential of an exercise stimulus (how much growth we can expect over time). Although many studies have examined performance measures across the menstrual cycle, only a handful of studies to examine the changes in muscle thickness. A study done by Wong et al (38), looked at the influence of biological sex and cuff width on muscle swelling, echo intensity, and the fatigue response to blood flow restricted exercise. Although they did not account for phase in the cycle, authors noted (similar to the present findings) that men overall swelled more than women. Despite this, there were not significant differences between sexes for muscle swelling when expressed as a percentage change. When examining long term growth, Sakamaki-Sunaga and colleagues observed that after 12 weeks of resistance training, the variations in female hormones induced by the menstrual cycle changes of eumenorrheic women do not significantly influence muscle hypertrophy or strength adaptations when different training frequencies are used (34). Results of the present study would suggest that phase of the menstrual cycle does not impact acute muscle swelling. Despite these, there seems to be a lack of data regarding the influence of the menstrual cycle on long term outcomes of resistance training. We would suggest that more research be conducted on muscular hypertrophy and muscle swelling throughout the menstrual cycle in females. 


\section{Total Body Water}

Some studies have observed fluctuations in total body water composition throughout the different phases of the menstrual cycle. Tomazo- Ravnik and colleagues recorded bioelectrical impedance measurements during two consecutive menstrual cycles, they found that both body mass and total body water parameters were highest in the luteal phase and lowest in the follicular phase in $65 \%$ of the young women in their sample (40 women overall) (39). These results are dissimilar to the present study. The present study observed no changes in total body water throughout the menstrual cycle over time for either sex. However, the present findings are in line with those of McKee and Cameron whom observed no changes in total body water, via bioelectrical impedance, or body weight in women taking birth control and women not taking birth control, as well as a male control group, over the different phases of the menstrual cycle (40). The primary purpose of including total body water in the present study was to determine if changes in body water may help explain changes in muscle thickness or acute swelling across the mensural cycle. Our findings suggest that none of these measures appear to change appreciably across the menstrual cycle when compared to a time-matched control group of men.

\section{Limitations}

This present study is not without limitation. For example, levels of hormones were not tested throughout the different phases of the menstrual cycle. However, the tracking app and at home ovulation kits were used to confirm whether women were ovulating. In addition, muscle swelling was inferred based off of acute changes in ultrasound muscle thickness measures. As such, we are not able to actually measure if the fluid was shifting into the muscle cells or just into the 
interstitial space. However, this technique has been used previously as a surrogate for muscle swelling (7).

\section{Conclusions}

Results of the present study suggest that the menstrual cycle may have little influence on muscle thickness, acute changes in muscle thickness (swelling), total body water or isometric strength when compared to a group of time-matched male controls. These findings suggest that studies examining acute changes in skeletal muscle may not need to account for presence of the menstrual cycle. Although the present study did not measure long-term adaptations to exercise, the similar acute response observed across the menstrual cycle may suggest that phase of the cycle may not impact long term adaptations of muscle size and strength. However, future studies examining long term and chronic adaptation are needed to better understand the influence of the menstrual cycle on muscle adaptation. 


\section{References}

1. Buckner SL, Jessee MB, Dankel SJ et al. Blood Flow Restriction Does Not Augment Low Force Contractions Taken to or Near Task Failure: 3521: Board\# 209 June 1 9: 30 AM- 11: 00 AM. Medicine \& Science in Sports \& Exercise. 2019;51(6):9701.

2. Counts BR, Buckner SL, Dankel SJ et al. The acute and chronic effects of "NO LOAD” resistance training. Physiology \& Behavior. 2016.

3. Jessee M, Buckner S, Mouser JG et al. Muscle adaptations to high-load training and very low- load training with and without blood flow restriction. Frontiers in physiology. $2018 ; 9: 1448$.

4. Dupont AC, Sauerbrei EE, Fenton PV, Shragge PC, Loeb GE, Richmond FJ. Realtime sonography to estimate muscle thickness: comparison with MRI and CT. J Clin Ultrasound. 2001;29(4):230-6.

5. Damas F, Phillips SM, Lixandrao ME et al. Early resistance training-induced increases in muscle cross-sectional area are concomitant with edema-induced muscle swelling. Eur J Appl Physiol. 2015.

6. Yitzchaki N, Kuehne TE, Mouser JG, Buckner SL. Can changes in echo intensity be used to detect the presence of acute muscle swelling? Physiological measurement. 2019;40(4):045002. 
7. Buckner SL, Dankel SJ, Mattocks KT et al. Differentiating swelling and hypertrophy through indirect assessment of muscle damage in untrained men following repeated bouts of resistance exercise. Eur J Appl Physiol. 2017;117(1):213-24.

8. Damas F, Phillips SM, Lixandrao ME et al. Early resistance training-induced increases in muscle cross-sectional area are concomitant with edema-induced muscle swelling. Eur $J$ Appl Physiol. 2016;116(1):49-56.

9. Phillips S, Sanderson A, Birch K, Bruce S, Woledge R. Changes in maximal voluntary force of human adductor pollicis muscle during the menstrual cycle. The Journal of physiology. 1996;496(2):551-7.

10. Sarwar R, Niclos BB, Rutherford OM. Changes in muscle strength, relaxation rate and fatiguability during the human menstrual cycle. $J$ Physiol. 1996;493 ( Pt 1):267-72.

11. Watson PE, Robinson MF. Variations in Body-Weight of Young Women during the Menstrual Cycle. Br J Nutr. 1965; 19:237-48.

12. Elliott K, Cable N, Reilly T, Diver M. Effect of menstrual cycle phase on the concentration of bioavailable 17- $\beta$ oestradiol and testosterone and muscle strength. Clinical science. 2003;105(6):663-9.

13. Fox 3rd S, Haskell W. Physical activity and the prevention of coronary heart disease. Bulletin of the New York Academy of Medicine. 1968;44(8):950.

14. Howley ET. You asked for it Question Authority. ACSM's Health \& Fitness Journal. 2000;4(4):6-18.

15. Counts BR, Rossow LM, Mattocks KT et al. Let's talk about sex: where are the young females in blood flow restriction research? Clinical physiology and functional imaging. 2018;38(1):1-3. 
16. Sakamaki M, Yasuda T, Abe T. Comparison of low-intensity blood flow-restricted traininginduced muscular hypertrophy in eumenorrheic women in the follicular phase and luteal phase and age-matched men. Clinical physiology and functional imaging. 2012;32(3):185-91.

17. De Jonge XJ, Boot C, Thom J, Ruell P, Thompson M. The influence of menstrual cycle phase on skeletal muscle contractile characteristics in humans. The Journal of physiology. $2001 ; 530(1): 161-6$.

18. Lebrun CM. Effect of the different phases of the menstrual cycle and oral contraceptives on athletic performance. Sports medicine. 1993;16(6):400-30.

19. Lebrun CM, McKenzie DC, Prior JC, Taunton JE. Effects of menstrual cycle phase on athletic performance. Medicine and Science in Sports and Exercise. 1995;27(3):437-44.

20. DiBrezzo R, Fort IL, Brown B. Relationships among strength, endurance, weight and body fat during three phases of the menstrual cycle. The Journal of sports medicine and physical fitness. 1991;31(1):89-94.

21. Quadagno D, Faquin L, Lim G-N, Kuminka W, Moffatt R. The menstrual cycle: does it affect athletic performance? The Physician and sports medicine. 1991;19(3):121-4.

22. Gür H. Concentric and eccentric isokinetic measurements in knee muscles during the menstrual cycle: a special reference to reciprocal moment ratios. Archives of physical medicine and rehabilitation. 1997;78(5):501-5.

23. Rutherford O, Jones D, Newham D. Clinical and experimental application of the percutaneous twitch superimposition technique for the study of human muscle activation. Journal of neurology, neurosurgery \& psychiatry. 1986;49(11):1288-91. 
24. Prior JC, Vigna Y, Sciarretta D, Alojado N, Schulzer M. Conditioning exercise decreases premenstrual symptoms: a prospective, controlled 6-month trial. Fertility and Sterility. 1987;47(3):402-8.

25. Horvath SM, Drinkwater BL. Thermoregulation and the menstrual cycle. Aviat Space Environ Med. 1982;53(8):790-4.

26. De MS, Maguire MS, Rubin KR, Maresh CM. Effects of menstrual phase and amenorrhea on exercise performance in runners. Medicine and science in sports and exercise. 1990;22(5):575-80.

27. Janse de Jonge XA. Effects of the menstrual cycle on exercise performance. Sports Med. 2003;33(11):833-51.

28. Ishida Y, Carroll J, Pollock M, Graves J, Leggett S. Reliability of B-mode ultrasound for the measurement of body fat and muscle thickness. American journal of human biology. 1992;4(4):511-20.

29. Haussinger D, Roth E, Lang F, Gerok W. Cellular hydration state: an important determinant of protein catabolism in health and disease. Lancet. 1993;341(8856):1330-2.

30. Haussinger D, Gerok W. Role of the cellular hydration state for cellular function: physiological and pathophysiological aspects. Advances in experimental medicine and biology. 1994; 368:33-44.

31. Buckner SL, Dankel SJ, Counts BR et al. Influence of cuff material on blood flow restriction stimulus in the upper body. J Physiol Sci. 2016.

32. Buckner SL, Jessee MB, Dankel SJ et al. Acute skeletal muscle responses to very low-load resistance exercise with and without the application of blood flow restriction in the upper body. Clinical physiology and functional imaging. 2018. 
33. Yasuda T, Loenneke JP, Thiebaud RS, Abe T. Effects of blood flow restricted low-intensity concentric or eccentric training on muscle size and strength. PLoS One. 2012;7(12): e52843.

34. Sakamaki-Sunaga M, Min, S, Kamemoto K, Okamoto T. Effects of Menstrual-Phase Dependent Resistance Training Frequency on Muscular Hypertrophy and Strength. Journal of Strength and Conditioning Research. 2016;30(6):1727-1734.

35. Arazi H, Nasiri S, Eghbali E. Is there a difference toward strength, muscular endurance, anaerbic power and hormonal changes between the three phases of the menstrual cycle of active girls? Apunts. 2019;54(202):65-72.

36. Ekenros L, Hirschberg AL, Heijne A, Friden C. Oral Contraceptives Do Not Affect Muscle Strength and Hop Performance in Active Women. Clinical Journal or Sport Medicine. 2013;23(3):202-207.

37. Giacomoni M, Bernard T, Gavarry O, Altare S, Falgairette G. Influence of the menstrual cycle phase and menstrual symptoms on maximal anaerobic performance. Med Sci Sports Exerc. 2000;32(2):486-492.

38. Wong V, Abe T, Chatakondi RN, Bell ZW, Spitz RW, Dankel SJ, Loenneke JP. The influence of biological sex and cuff width on muscle swelling, echo intensity, and the fatigue response to blood flow restricted exercise. Journal of Sports Sciences. 2019;37(16).

39. Tomazo-Ravnik T, Jakopic V. Changes in Total Body Water and Body Fat in Young Women in the Course of Menstrual Cycle. International Journal of Anthropology. 2006; 21:55-60 40. McKee JE, Cameron N. Bioelectrical impedance changes during the menstrual cycle. American Journal of Human Biology. 1998;9(2): 155-161. 
41. Kuehne TE, Yitzchaki N, Jessee MB, Graves BS, Buckner SL. A comparison of acute changes in muscle thickness between A-mode and B-mode ultrasound. Phys Measurement. 2019;40(11) 
Appendices 


\section{Appendix A}

\section{Exclusionary Questionnaire Checklist}

\begin{tabular}{|l|c|c|}
\hline & Yes & No \\
\hline Resistance trained in biceps curls for the last 6 months? & & \\
\hline Exercised in the last 24 hours? & & \\
\hline Younger than 18 years old? & & \\
\hline Older than 35 years old? & & \\
\hline Use tobacco? & & \\
\hline Major surgery within the last six months? & & \\
\hline Orthopedic problems preventing strength testing/exercise. & & \\
\hline On Hypertensive Medication? & & \\
\hline Heavy Alcohol Consumption in the last 24 hours & & \\
\hline Known reproductive and/or endocrine disorder & & \\
\hline $\begin{array}{l}\text { Irregular menstrual cycle (including amenorrhea, or missed } \\
\text { periods) }\end{array}$ & & \\
\hline Pregnant or planning to become pregnant & & \\
\hline
\end{tabular}




\section{Appendix B}

\section{Physical Activity Readiness Questionnaire}

\section{$=\quad$ PAR-Q \& YOU \\ (A Questionnaire for People Aged 15 to 69)}

Regular physical activity is fun and healthy, and increasingly more people are starting to become more active every day. Being more active is very safe for most people. However, some people should check with their doctor before they start becoming much more physically active.

If you are planning to become much more physically active than you are now, start by answering the seven questions in the box below. If you are between the ages of 15 and 69 , the PAR-Q will tell you if you should check with your doctor before you start. If you are over 69 years of age, and you are not used to being very active, check with your doctor.

Common sense is your best guide when you answer these questions. Please read the questions carefully and answer each one honestly: check YES or NO.

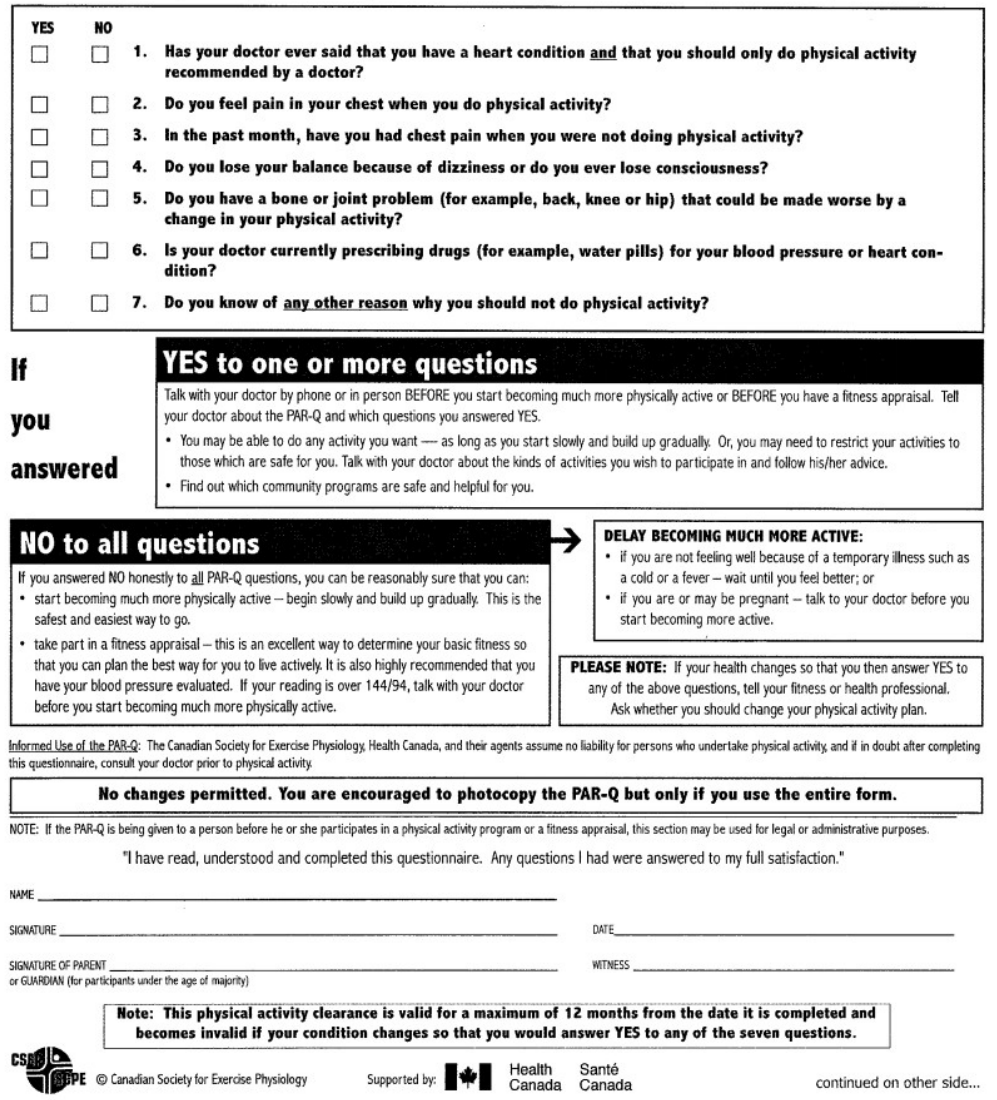

